\title{
Hybrid TB-LEACH Energy Based Multihop Protocol
}

\author{
Gaganpreet Kaur \\ Dept. of Computer Science and Engineering, \\ Guru Nanak Dev University, \\ Amritsar
}

\author{
Sandeep Waraich \\ Dept. of Computer Science and Engineering, \\ Guru Nanak Dev University, \\ Amritsar
}

\begin{abstract}
Wireless sensor network is defined as the network composed of tiny and energy restricted sensor nodes. Sensor nodes collect the useful information from surrounding environment and deliver it to the base station. Sensor nodes have limited energy. Routing protocols must consume minimum energy in order to prolong the network lifetime. Clustering is most energy efficient technique. LEACH is one of the fundamental clustering based protocols. Many researchers had proposed changes in the LEACH protocol to overcome the drawbacks of LEACH protocol. In this paper, a hybrid protocol based on two extensions of LEACH protocol named TB-LEACH and CTPEDCA protocol has been proposed. This hybrid protocol also considers two additional factors residual energy of sensor nodes and distance of cluster head nodes from the base station. The performance of LEACH, TB-LEACH, CTPEDCA and proposed protocol is compared on the basis of parameters FND (First node die time), HND (Half Node die time), LND (Last Node die time) and remaining energy per round.
\end{abstract}

\section{Keywords}

Sensor nodes; LEACH; TB-LEACH; CTPEDCA; FND; HND; LND

\section{INTRODUCTION}

Wireless sensor network consists of hundreds or even thousands of tiny energy restricted sensor nodes which are disseminated in a geographical region for monitoring of the physical or environmental conditions such as pressure, temperature etc. [2], [3], [12]

Each Sensor node is outfitted with one or more sensing elements, a data processor, a power source, communicating components and an energy source called battery. Sensing elements sense the surrounding conditions and perform measurements of these conditions. These measurements are converted into electrical signals and processed by the data processor. Then the communicating components of the sensors send this data to the base station. Sensor nodes have resource constraints such as limited energy, little storage capacity etc. As in most of the applications sensor nodes are deployed in unattended environment so battery of sensor nodes cannot be replaced or recharged. So, the routing protocols must save the battery of sensor nodes which prolongs lifespan of the network. [4], [5]

Most of the WSNs applications demand that only the data of user interest must be sent to the base station, unlike other wireless networks, in sensor network it is not essential to send all the sensed data to the BS. [9]

In routing protocols which work on the principle of clustering, sensor nodes are grouped together into clusters. Each cluster contains a coordinating node called cluster head and member nodes. Sensor nodes send their data to cluster heads instead of base station, and cluster heads perform aggregation of received data and transmit it further to the base station. [5], [6], [8]

Data aggregation conserves energy and diminishes the quantity of data transmitted to the Base station by accumulating the data at intermediate nodes and by forwarding towards the sink node only the result of an aggregation operation. One such energy efficient protocol which has combined data fusion and routing is LEACH (Low Energy Adaptive Clustering Hierarchy) protocol. [9]

LEACH is well known clustering based routing protocol. LEACH provides equal chance to all sensor nodes to become cluster head by incorporating rotation of the cluster head positions among nodes and by randomly selecting the cluster head. LEACH has prolonged the lifetime of the network and balanced the consumption of energy as compare to non clustering protocols. [5], [6], [7]

The main focus of this paper is on increasing network lifetime by making most energy efficient nodes as cluster heads. In this paper a hybrid energy efficient protocol has been proposed by combining the two extensions of LEACH protocol named TB-LEACH and CTPEDCA (A cluster-based and tree-based power efficient data collection and aggregation protocol for wireless sensor networks) and two additional factors residual energy of nodes and distance of cluster head nodes from the base station are also considered.

\section{RELATED WORK}

In [6] authors have proposed LEACH protocol which is based on clustering. In this protocol, energy load is distributed by rotating cluster head role among the nodes. In each round of LEACH protocol, there are two phases: First phase is Set-up phase and second phase is Steady State phase.

\section{Set-up Phase:}

In set-up phase nodes make a decision regarding whether to act as cluster-head or not for the current round. This decision is taken by nodes based on the value of $\mathrm{P}$ (Probability for becoming the cluster head) and how many times the node has previously acted as a cluster-head. For this at the starting of each round a random number is chosen by each node which lies between 0 and 1 . If the number chosen by the node is less than the threshold value $T(n)$ then that node becomes the cluster-head for that round. The formula used for calculating threshold is given below:

$$
T(n)= \begin{cases}\frac{P}{1-P\left(r \bmod \frac{1}{P}\right)} & \text { if } n \in G \\ 0 & \text { otherwise }\end{cases}
$$

Where $\mathrm{P}$ stands for the desired percentage of cluster heads, $r$ represents the current round number, and $\mathrm{G}$ is the set which 
contains all the nodes that have not acted as cluster-heads in the last 1/P rounds. The threshold makes sure that each node act as a cluster-head once within 1/P rounds. Each node which is elected as a cluster head broadcasts cluster head advertisement message using a CSMA MAC protocol.

After this each sensor node other than cluster head nodes decides to which cluster it will belong and inform that cluster head by sending a join request message.

On the basis of no. of cluster member nodes, each cluster head creates a TDMA schedule and broadcast it to the member nodes which contain the information about the time slot when any node can transmit.

\section{Steady State phase}

In Steady state phase each node sends its data to its own respective cluster head during its allocated time slot. After receiving the data from the cluster members, cluster head nodes aggregate it and send this data to base station.

In [2] authors have proposed an improvement over LEACH protocol called TB-LEACH protocol. TB-LEACH protocol has improved the cluster head selection criteria of LEACH protocol for improving the partitions of clusters. In this protocol in each round constant number of cluster heads are elected on the basis of random timer. A counter is used for electing constant number of cluster heads. At the starting of each round every node generates a random timer and back off it. When the timer of a node got expired, it checks the no. of received cluster head messages, if it is less than four then it declares itself as a cluster head and the rest of the process is same as LEACH protocol.

In [1] authors have proposed LEACH-B protocol. LEACH-B protocol elects constant number of nodes as cluster heads in each round. In this protocol cluster head selection is carried out in two steps. In first step cluster heads selection is performed according to LEACH protocol, so uncertain number of cluster heads are obtained and second selection is performed to make the number of cluster heads constant. During second selection node's residual energy is considered. Some of lower energy cluster heads are transformed into normal nodes if number of nodes elected as cluster heads are higher than the constant number, and some of higher energy normal nodes are transformed into cluster heads if number of nodes elected as cluster heads are lower than constant number.

In [8] authors have proposed a protocol named CTPEDCA which is based on spanning tree and clustering. The cluster head selection criterion of this protocol is same as LEACH protocol. After the selection of cluster heads, clusters are formed and the selected cluster heads construct a spanning tree. The cluster head node with highest residual energy is elected as root node of the spanning tree. The cluster heads send aggregated data along the tree and finally the root node send data to the Base station. Only the root node uses the multipath fading channel model and other use free space channel models. It has prolonged the network life time by reducing energy consumption.

In [4] authors have proposed E-LEACH protocol. In this protocol criterion of selection of cluster heads is improved by considering the initial energy and residual energy of the sensor nodes. At beginning of each round, round time is calculated. Cluster heads form a spanning tree among them and send their data to the base station along this tree.
In [5] authors have proposed LEACH-R protocol. In this LEACH-R protocol cluster heads are selected by considering the residual energy of the nodes. With this modification probability of the sensor nodes having more remaining energy increases to take the role of cluster head. In each round one relay node is chosen out of the selected cluster heads. Relay node is selected on basis of residual energy of cluster heads and their distances from the base station. Cluster heads after collecting the data from local clusters send this to the relay node. The relay node finally delivers this data to the base station.

In [13] authors have proposed I-LEACH. I-LEACH protocol has three phases. During cluster head selection phase this protocol has modified the threshold value by considering various properties of sensor nodes such as their current energy level, number of neighbor nodes, and their distances to base station. It also considers average energy of the network, average number of neighbor nodes in the network, average distances of nodes from base station. In cluster formation phase nodes take into account the distances of cluster heads from the base station. In Data transmission phase cluster heads transmit data to the base station after collecting data from their local cluster members. Nodes which are very nearer to base station they are allowed to transmit data to the base station directly, it has reduced extra transfers. This protocol has reduced the average consumed energy and prolonged the WSN lifetime.

\section{LIMITATIONS IN EXISTING PROTOCOLS}

1. In LEACH and CTPEDCA protocols, in each round different number of nodes are chosen as cluster heads i.e. in each round number of cluster heads are not constant.

2. In LEACH and TB-LEACH protocols, all the cluster head nodes transfer their data to the base station in a single-hop manner.

3. In LEACH, TB-LEACH and CTPEDCA protocols energy of the sensors nodes is not taken into consideration while selecting the cluster heads.

4. In CTPEDCA protocol during selection of the root node of the minimum spanning tree the distance of cluster heads from base station is not taken into consideration.

\section{PROPOSED PROTOCOL}

As the basic criteria of LEACH protocol this proposed protocol is also broken into rounds. In the Proposed protocol constant number of nodes are elected as cluster heads in each round by using a timer which is based on residual energy of the sensor nodes. The cluster heads send their data to the base station by the means of minimum spanning tree. During cluster head selection residual energy of sensor nodes is considered so the nodes having more residual energy get more chance to become the cluster head. By making use of minimum spanning tree only one node has to communicate with the far distant base station. If the network has $\mathrm{k}$ clusters only one node has to use multipath fading channels model and $\mathrm{k}-1$ use free space channel model which significantly reduces the consumed energy. Each round of proposed protocol has four phases: Selection of Cluster heads, Formation of Clusters, Formation of minimum spanning Tree and Data transmission. The flow chart of the proposed protocol is as shown in Figure 1. 


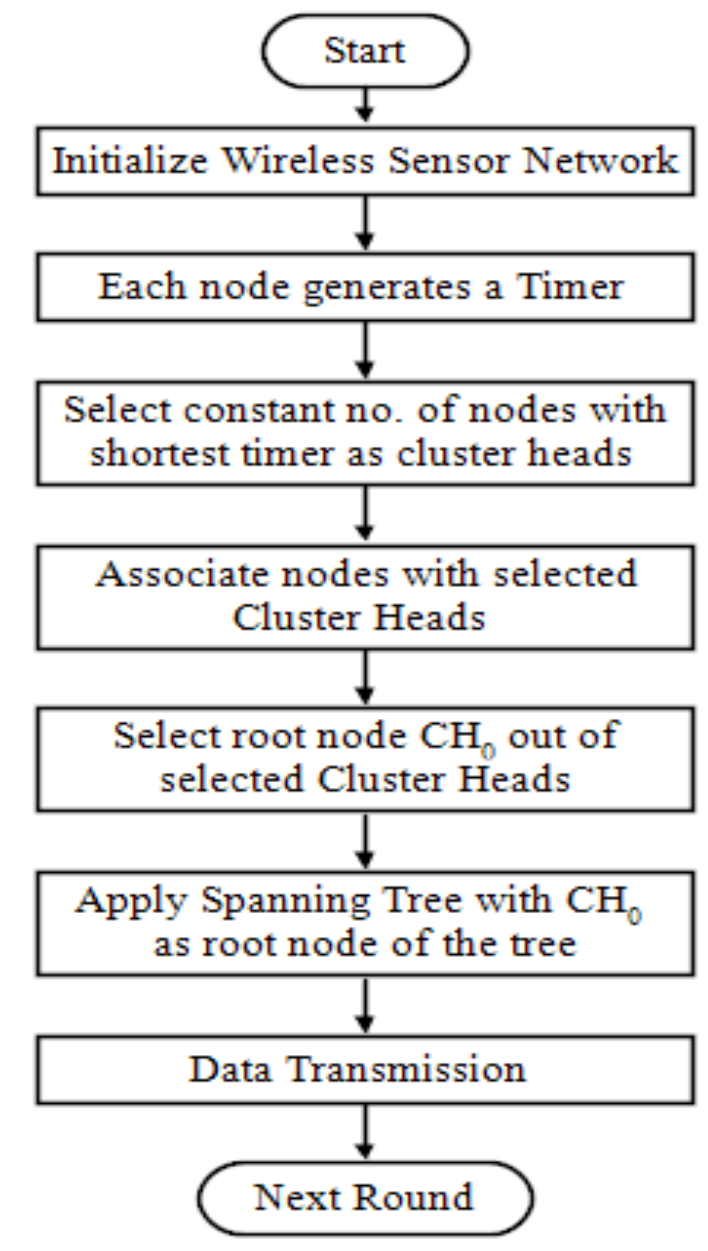

Fig1: Flowchart of Proposed Protocol

The detail of each phase is given below.

\section{Phase 1: Selection of Cluster Heads}

A timer is generated by all the nodes and competition for becoming cluster head among the sensor nodes depend on this timer. The nodes having the smaller timer win the competition and become cluster heads. For the purpose that there is always a constant number of cluster-heads, a counter is used. When the limit of the counter is reached the specified value, node's competition for becoming cluster-heads is no longer carried on. In TB-LEACH in each round random timer is chosen by nodes. In TB-LEACH residual energy of the nodes is not considered while assigning the timer. In proposed protocol timer chosen by nodes depend on their residual energy. Initially all the nodes have same initial energy so for first round all the nodes choose the random timer and after first round the timer chosen by the nodes depend on their residual energy. The timer chosen by nodes is given as

$$
\operatorname{Timer}(\mathrm{i})=\left\{\begin{array}{lr}
\text { randi }(\text { maxlimit, } 1,1) & \text { if } \mathrm{r}=0 \\
\frac{\mathrm{K}}{\text { residualenergy(i) }} & \text { otherwise }
\end{array}\right.
$$

Where $\mathrm{r}$ represents round number, $\mathrm{i}$ represents id of sensor node, and $\mathrm{K}$ is a positive integer, initially $\mathrm{K}$ is given some highest value. It is dynamically modified by the root node in phase 3 .
The timer chosen by the sensor nodes depend on their residual energy, so the nodes having higher residual energy get shortest timer and have more chance elect as cluster heads. When timer of any node expires it will check no. of advertisement messages received so far if it is less than the limit of the counter it will announce its cluster head status by broadcasting advertisement message using non persistent CSMA MAC protocol otherwise it cannot become cluster head for the current round.

\section{Phase 2: Formation of Clusters}

All cluster-heads broadcast their advertisement messages using the same transmit energy. After receiving advertisement messages each sensor node makes a decision to which cluster it will belong and the nodes decide on the basis of the received signal strength of the advertisement messages. When each node has made its decision to which cluster it will belong, it inform that cluster head by sending a join-request message (Join-REQ) using non persistent CSMA MAC protocol. Each cluster head node sets up a TDMA schedule and transmits this schedule to its own cluster members.

Phase 3: Formation of Minimum Spanning Tree

After formation of clusters all the selected cluster heads broadcast their advertisement messages containing information such as id of cluster head, id of cluster, information of location, cluster size, a parameter ED which is equal to the ratio of their residual energy and their distance from the base station. Each cluster head node receives and save this information. Finally each cluster head get a table which contains information of all cluster heads. Then the cluster head having highest value of ED (residual energy divided by distance of cluster head node from the base station) [5] parameter is selected as root node of the tree. Then the function for creating minimum spanning tree is called which is implemented by root node. After creation of minimum spanning tree the root node broadcasts the tree information message to other cluster heads. This message contain data transmission schedule among the cluster heads. The root node also modifies the value of $\mathrm{K}$ as:

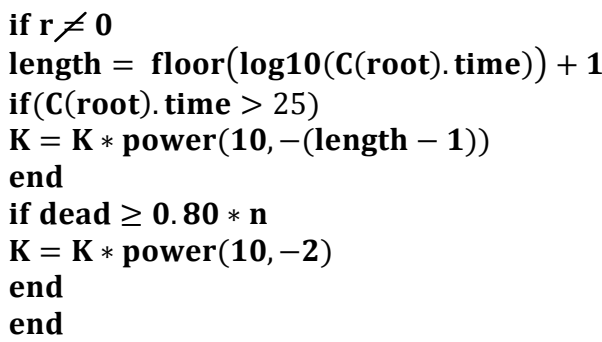

Where $r$ represents round number, root represents the root node of the spanning tree, $\mathrm{n}$ is the total number of nodes in the network.

In each round except first round root node checks two conditions.

Condition 1: Root node checks the timer assigned to it during that round if it is greater than 25 then it finds the length of time allotted to it (no. of digits in the time allotted to it). It multiplies the value of $\mathrm{K}$ by $10^{- \text {(length- } 1)}$.

Condition 2: Root node also checks for the no. of dead nodes. If $80 \%$ or above $80 \%$ of the nodes are dead then it multiplies the value of $\mathrm{K}$ by $10^{-2}$.

If any of the above condition met root node modifies the value of $\mathrm{K}$. The value of the $\mathrm{K}$ is modified according to above these two conditions so that the waiting timer does not go very high. Root node broadcasts this value to other cluster heads. After 
receiving from root node, cluster heads broadcast this value of $\mathrm{K}$ to their cluster members.

\section{Phase 4: Data transmission}

In Data transmission phase non cluster head nodes send their data to their respective cluster head node during their allocated time slot. Once the cluster head nodes have received data from their cluster members, they aggregate this data and generate a useful packet. When all cluster heads have completed collection and aggregation of data from their local cluster nodes, they send their data along the tree. Each receiving cluster head performs data aggregation of the local cluster data and data received from the previous cluster head in the tree before transmitting it to next cluster head. Final resultant data is sent by root node to the base station.

\section{SIMULATION ANALYSIS}

In order to evaluate the performance of proposed protocol, LEACH, TB-LEACH, CTPEDCA and proposed protocols are simulated using MATLAB. The performance is evaluated on the basis

(i) Dead Node Analysis on the basis of metrics FND (round at which first node dies), HND (round at which half nodes die), and LND (round at which last node die).

(ii) Remaining Energy per round.

\subsection{Network Model}

The network area of $100 \times 100 \mathrm{~m}^{2}$ is considered and 100 nodes are randomly deployed in the area. The Base station is located outside the region. Base station and sensor nodes are fixed. All the sensor nodes in the network are homogeneous and all nodes have limited amount of energy.

\subsection{Simulation Parameters}

The parameter values which have been used for simulation are listed below in the Table 1 .

TABLE 1. Table of Values of the Parameters

\begin{tabular}{|c|c|}
\hline Parameters & Values \\
\hline Number of Sensors & 100 \\
\hline Network Dimensions & $100 \mathrm{~m} \times 100 \mathrm{~m}$ \\
\hline Initial Energy & $0.5 \mathrm{~J}$ \\
\hline Transmitter Electronics $\left(\mathrm{E}_{\mathrm{elec}}\right)$ & $50 \mathrm{~nJ} / \mathrm{bit}$ \\
\hline Receiver Electronics $\left(\mathrm{E}_{\text {elec }}\right)$ & $50 \mathrm{~nJ} / \mathrm{bit}$ \\
\hline $\mathrm{E}_{\mathrm{fs}}$ & $10 \mathrm{pJ} / \mathrm{bit} / \mathrm{m}^{2}$ \\
\hline $\mathrm{E}_{\mathrm{mp}}$ & $0.0013 \mathrm{pJ} / \mathrm{bit} / \mathrm{m}^{4}$ \\
\hline $\mathrm{E}_{\mathrm{DA}}$ & $5 \mathrm{~nJ} / \mathrm{bit} / \mathrm{signal}$ \\
\hline $\mathrm{d}_{0}$ & $\mathrm{Sqrt}\left(\mathrm{E}_{\mathrm{fs}} / \mathrm{E}_{\mathrm{mp}}\right)$ \\
\hline Data Packet length & $4200 \mathrm{bits}$ \\
\hline Base station & $(50,250)$ \\
\hline $\mathrm{P}$ & 0.04 \\
\hline Initial Value of $\mathrm{K}$ & 1 \\
\hline
\end{tabular}

\subsection{Energy Consumption Model}

LEACH first order energy model is used [4, 7]. Transmitting cost of 1-bit message depends on the distance between the sender and receiver and is given by the equation

$$
E_{T x}(l, d)= \begin{cases}l E_{\text {elec }}+l E_{f s} d^{2} & \text { if } d<d_{o} \\ l E_{\text {elec }}+l E_{m p} d^{4} & \text { if } d \geq d_{o}\end{cases}
$$

Where $d$ is the distance between sender and receiver, $d_{o}$ is threshold distance, when $d$ is less than $d_{o}$ then free space model is used, otherwise multipath fading model is used. Receiving cost of 1-bit message is given by

$$
\mathrm{E}_{\mathrm{Rx}}(\mathrm{l})=\mathrm{lE}_{\text {elec }}
$$

The electronics energy $E_{\text {elec }}$ depends on factors such as the digital coding, modulation, filtering, and spreading of the signal whereas the amplifier energy $E_{\mathrm{fs}} \mathrm{d}^{2}$, or $\mathrm{E}_{\mathrm{mp}} \mathrm{d}^{4}$ depends on the distance to the receiver and the acceptable bit-error rate.

\subsection{Simulation Results}

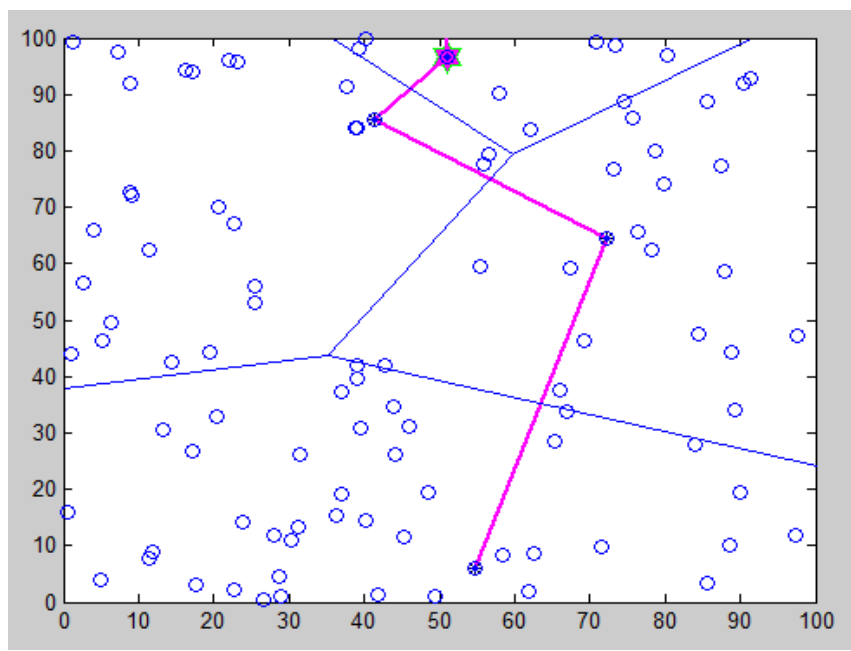

Fig 2: Sensor nodes deployed in network area of $100 \times 100 \mathrm{~m}^{2}$

Fig. 2 shows that 100 nodes are randomly deployed in the network are of $100 \times 100 \mathrm{~m}^{2}$. The four cluster heads are elected in each round. The normal nodes are represented by symbol $(\mathrm{O})$ and cluster head nodes are represented by symbol ( 3 ). Different cluster partitions are shown by the blue lines. A spanning tree is created among the cluster heads shown by magenta lines. The root node of the spanning tree is represented by symbol ( $/$ ase station is not shown in the fig. because base station is located outside the region. The parameters used for simulation are listed in the above Table 1 .

\subsubsection{Dead Node Analysis}

The Figure 3 compares the number of dead nodes at each round in $\mathrm{LEACH}, \mathrm{TB}-\mathrm{LEACH}, \mathrm{CTPEDCA}$ and proposed protocol.

The values of FND (round at which first node dies), HND (round at which half nodes die), and LND (round at which last node die) of all the four protocols are given below in the Table 2.

TABLE 2. FND, HND AND LND Comparison

\begin{tabular}{|c|c|c|c|}
\hline Protocols & FND & HND & LND \\
\hline LEACH & 301 & 469 & 574 \\
\hline TB-LEACH & 393 & 545 & 769 \\
\hline CTPEDCA & 593 & 698 & 759 \\
\hline Proposed & 867 & 907 & 914 \\
\hline
\end{tabular}


FND: FND refers to the round number at which first node of the network runs out of energy. The period from the $1^{\text {st }}$ round to the round number at which first node dies is called stability period. Network becomes unstable after the death of first node. $[10,11]$
It is clear from the table 2 and Figure 3 that first node dies later in the proposed protocol than the other three protocols. FND of the proposed protocol is approximately 2.9 times of LEACH, 2.2 times of TB-LEACH and 1.5 times of CTPEDCA.

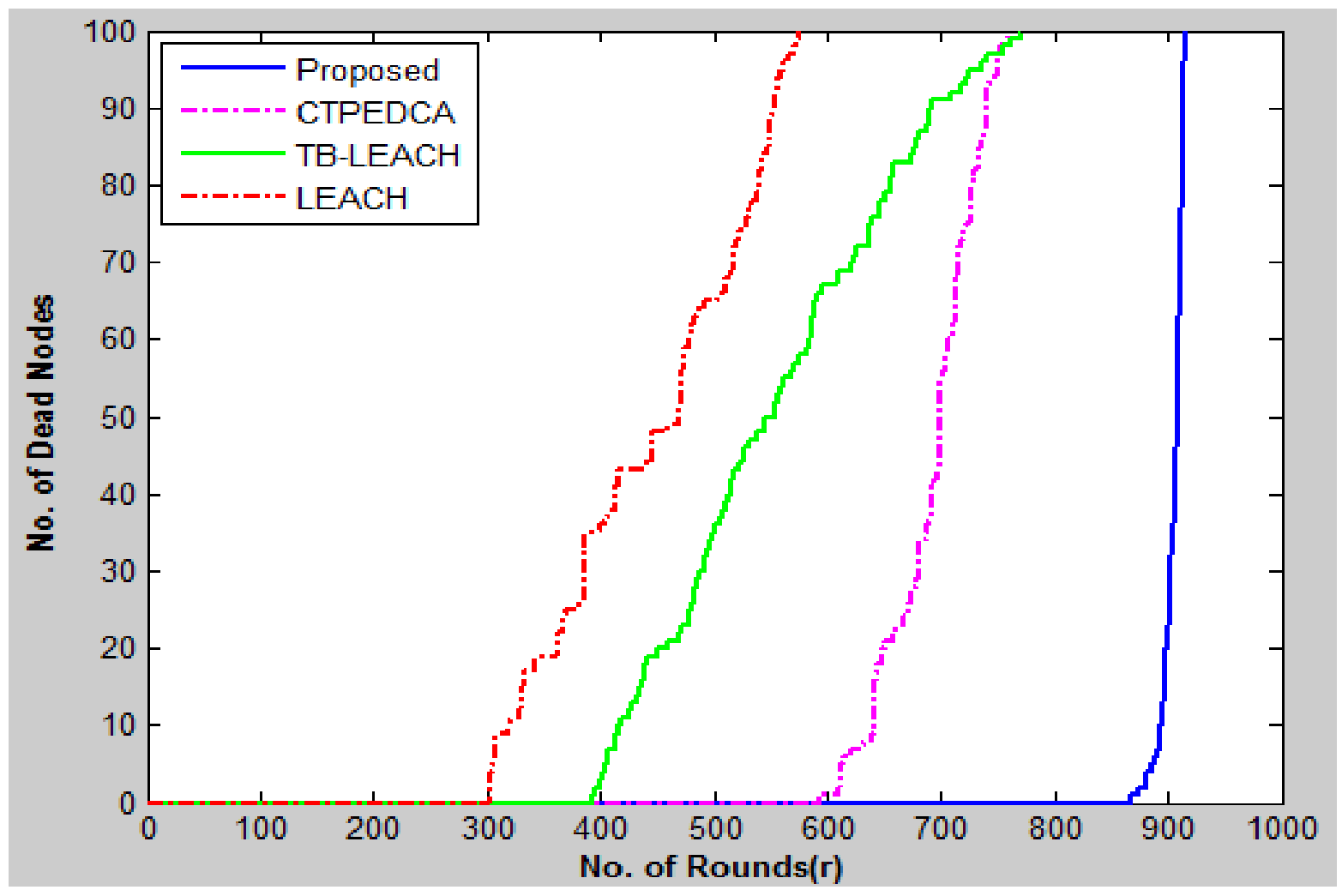

Fig 3: Dead node Comparison of LEACH, TB-LEACH, CTPEDCA and Proposed Protocol

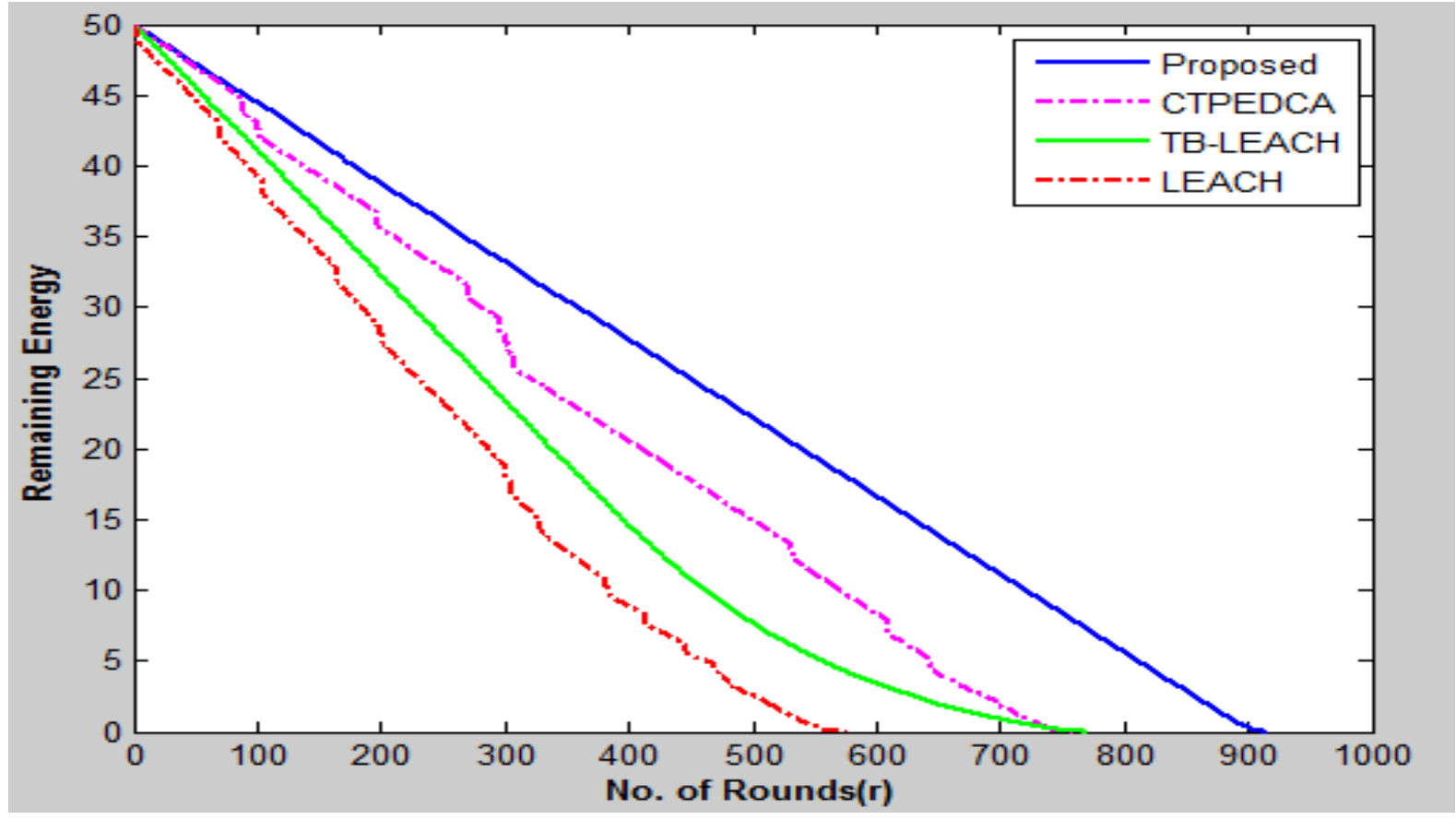

Fig 4: Remaining Energy Comparison of LEACH, TB-LEACH, CTPEDCA and Proposed protocol 
Thus Stability period of proposed protocol is prolonger than LEACH, TB-LEACH and CTPEDCA protocols. CTPEDCA protocol is more stable than TB-LEACH and LEACH, while stability period of TB-LEACH is longer than LEACH protocol.

$H N D$ : HND refers to the round number at which half of the nodes of the network are dead. $[10,11]$

HND of proposed protocol is approximately 1.9 times of LEACH protocol, 1.7 times of TB-LEACH and 1.3 times CTPEDCA protocol. HND of CTPEDCA protocol is more than TB-LEACH and LEACH, while HND of TB-LEACH is more than LEACH protocol.

$L N D$ : LND refers to the round number at which last node of the network dies. LND represents the network lifetime. The period between FND and LND is called instability period. This period should as little as possible. $[10,11]$

The proposed protocol has improved the network life time approximately by $59 \%$ as compared to LEACH, by $19 \%$ as compared to TB-LEACH and by $20 \%$ as compared to CTPEDCA. The CTPEDCA and TB-LEACH protocols have improved the network life time as compare to LEACH protocol. The instability period of proposed protocol is smallest as compared to other three protocols.

\subsubsection{Remaining Energy Analysis}

Fig.4 shows performance of remaining energy of four protocols LEACH, TB-LEACH, CTPEDCA and Proposed protocols. Proposed protocol has more remaining energy at each round as compared to LEACH and TB-LEACH protocols. Remaining energy of proposed protocol and CTPEDCA is equal in beginning but after a certain period (near about 100 rounds in our simulation) proposed protocol has more remaining energy than CTPEDCA protocol. CTPEDCA and TB-LEACH protocols have more remaining energy than LEACH protocol.

\section{CONCLUSION}

In this paper a hybrid protocol based on TB-LEACH and CTPEDCA protocols has been proposed. The proposed protocol also considers the residual energy of sensor nodes and distance of sensor nodes from the base station. To evaluate the performance of proposed protocol, its performance is compared with LEACH, TB-LEACH and CTPEDCA protocols. The simulation results have shown that the proposed protocol has improved all the three parameters (FND, HND and LND) as compare to LEACH, TB-LEACH and CTPEDCA protocols. Thus proposed protocol has increased the stable region and network life time as compare to LEACH, TB-LEACH and CTPEDCA protocols. It is concluded that proposed protocol has outperformed the previous approaches LEACH, TB-LEACH, and CTPEDCA by consuming minimum amount of energy per round. In future performance of the proposed protocol will be analyzed with the change in the position of the base station.

\section{REFERENCES}

[1] Mu Tong, Minghao Tang, "LEACH-B: An Improved LEACH Protocol for Wireless Sensor Network", Wireless Communications Networking and Mobile Computing (WiCOM), International Conference on IEEE, 2010.
[2] Hu Junping, Jin Yuhui and Dou Liang, "A Time-based Cluster-Head election Algorithm for LEACH", $13^{\text {th }}$ IEEE Symposium on Computers and Communications(ISCC), pp. 1172-1176, 2008.

[3] M.Bani Yassein, A.Alzou'bi, Y.Khamayseh, and W.Mardini, "Improvement on LEACH Protocol of Wireless Sensor Network (VLEACH)", International Journal of Digital Content Technology and its Applications, vol. 3, no. 2, June 2009.

[4] Jia Xu, Ning Jin, Xizhong Lou, Ting Peng, Qian Zhou, and Yanmin Chen, "Improvement of LEACH protocol for WSN", Fuzzy Systems and Knowledge Discovery (FSKD), International Conference, IEEE, 2012.

[5] H.Srikanth.Kamath, "Energy Efficient Routing Protocol for Wireless Sensor Networks", International Journal of Advanced Computer Research, vol. 3, issue10, June 2013.

[6] Wendi B. Heinzelman, Anathan P. Chandraskan, and Hari Blakrisshnan, " Energy-Efficient Communication Protocol for Wireless Microsensor Networks", Proceedings of the 33rd Hawaii International Conference on System Sciences, IEEE, 2000.

[7] Wendi B. Heinzelman, Anathan P. Chandraskan, and Hari Blakrisshnan, "An Application-Specific Protocol Architecture for Wireless Microsensor Network", IEEE Trans. on Wireless Communications, vol. 1, issue 4, pp. 660-670, 2002

[8] Wei Wang, Bingwen Wang, Zhuo Liu, Lejiang Guo, and Wei Xiong, "A cluster-based and tree-based power efficient data collection and aggregation protocol for wireless sensor networks", Information Technology Journal,vol. 10, no. 3, pp. 557-564, 2011.

[9] Guangyan Huang, Xiaowei Li, and Jing He,"Dynamic Minimal Spanning Tree Routing Protocol for Large Wireless Sensor Networks", Industrial Electronics and Application (ICIEA), IEEE, 2006.

[10] Hu"seyin O" zgu"r Tan and I'brahim Ko"rpeog`lu, "Power Efficient Data Gathering and Aggregation Wireless Sensor Networks", SIGMOD Record, vol. 32, no. $4,2003$.

[11] Tripti Sharma, Dr.Brijesh Kumar, and Dr.Geetam Singh Tomar, "Performance Comparision of LEACH, SEP and DEEC Protocol in Wireless Sensor Network", Proc. of the International Conference on Advances in Computer Science and Electronics Engineering, 2012.

[12] Prabhat Kumar, M.P.Singh and U.S.Triar, "A Review of Routing Protocols in Wireless Sensor Network", International Journal of Engineering Research \& Technology (IJERT) vol. 1, issue 4, 2012.

[13] Zahra Beiranvand, Ahmad Patooghy, and Mahdi Fazeli, "I-LEACH: An efficient routing algorithm to improve performance \& to reduce energy co nsumption in Wireless Sensor Networks", $5^{\text {th }}$ IEEE Conference on Information and Knowledge Technology (IKT), pp.1318, 2013. 